PROCEEDINGS OF THE

AMERICAN MATHEMATICAL SOCIETY

Volume 42, Number 1, January 1974

\title{
HIGHER DERIVATIONS ON FINITELY GENERATED INTEGRAL DOMAINS
}

\author{
W. C. BROWN
}

\begin{abstract}
In this paper, we prove the following theorem: Let $A=k\left[x_{1}, \cdots, x_{t}\right]$ be a finitely generated integral domain over a field $k$ of characteristic zero. Then $A$ regular, i.e. the local ring $A_{q}$ is regular for all primes $q \subseteq A$, is equivalent to the following two conditions: (1) No nonminimal prime of $A$ is differential, and (2) $\operatorname{der}^{n}(A / k)=\operatorname{Der}^{n}(A / k)$ for all $n$. Here $\operatorname{Der}^{n}(A / k)$ denotes the $A$ module of all $n$th order derivations of $A$ into $A$ which are zero or $k$, and $\operatorname{der}^{n}(A / k)$ denotes the $A$-submodule of $\operatorname{Der}^{n}(A / k)$ generated by composites $\delta_{1} \circ \cdots \circ \delta_{j}(1 \leqq j \leqq n)$ of first order derivations $\delta_{i}$.
\end{abstract}

Introduction. Throughout this paper we assume $k$ is a field of characteristic zero. Let $A=k\left[x_{1}, \cdots, x_{t}\right]$ be a finitely generated integral domain over $k$. We shall let $\operatorname{Der}^{n}(A / k)$ denote the $A$-module of $n$th order derivations (see [6]) of $A$ to itself which vanish on $k$. It follows from Proposition 4 and Corollary 6.1 of [6] that any composite $\delta_{1} \circ \cdots \circ \delta_{j}(1 \leqq j \leqq n)$ of $j$-derivations $\delta_{i} \in \operatorname{Der}^{1}(A / k)$ is an $n$th order derivation in $\operatorname{Der}^{n}(A / k)$. The $A$-submodule of $\operatorname{Der}^{n}(A / k)$ spanned by all such composites will be denoted by $\operatorname{der}^{n}(A / k)$.

In general, one would like to know under what conditions is $\operatorname{der}^{n}(A / k)=$ $\operatorname{Der}^{n}(A / k)$ for all $n$. Recently K. Mount and O. E. Villamayor in [4] obtained a result for domains of dimension one over $k$. They proved the following result: Let $A$ be the coordinate ring of an irreducible algebraic curve over $k$. Let $p$ be a nonzero prime ideal of $A$. Then $A_{p}$ is a regular local ring if and only if $\operatorname{der}^{n}\left(A_{p} / k\right)=\operatorname{Der}^{n}\left(A_{p} / k\right)$ for all $n$. Let us say a finitely generated domain $A=k\left[x_{1}, \cdots, x_{t}\right]$ over $k$ is regular if $A_{q}$ is a regular local ring for all prime ideals $q \subseteq A$. Since $\operatorname{der}^{n}(A / k) \otimes_{A} A_{\circledast} \cong$ $\operatorname{der}^{n}\left(A_{q} / k\right)$ and $\operatorname{Der}^{n}(A / k) \otimes_{A} A_{q} \cong \operatorname{Der}^{n}\left(A_{q} / k\right)$, the result of Mount and Villamayor can be restated as follows: Let $A=k\left[x_{1}, \cdots, x_{t}\right]$ be a finitely generated integral domain over a field $k$ of characteristic zero. Suppose $A$ has dimension one over $k$, i.e., the quotient field of $A$ has transcendence degree one over $k$. Then $A$ is regular if and only if $\operatorname{der}^{n}(A / k)=\operatorname{Der}^{n}(A / k)$ for all $n$.

Received by the editors February 9, 1973.

AMS (MOS) subject classifications (1970). Primary 13 H05.

Key words and phrases. $n$th order derivation, $\operatorname{der}^{n}(A / k)$.

(c) American Mathematical Society 1974 
We shall say that a prime ideal $p \subseteq A$ is differential if $\delta(p) \subseteq p$ for all $\delta \in \operatorname{Der}^{1}(A / k)$. In this paper, we shall prove the following result: Let $A=k\left[x_{1}, \cdots, x_{t}\right]$ be a finitely generated integral domain over a field $k$ of characteristic zero. Then $A$ is regular if and only if (1) no nonminimal prime ideal $q \subseteq A$ is differential, and (2) $\operatorname{der}^{n}(A / k)=\operatorname{Der}^{n}(A / k)$ for all $n$.

Main results. We begin with the following fundamental theorem.

THEOREM 1. Let $A=k\left[x_{1}, \cdots, x_{t}\right]$ be a finitely generated integral domain over a field $k$ of characteristic zero. Then if (1) no nonminimal prime ideal $q \subseteq A$ is differential and (2) $\operatorname{der}^{n}(A / k)=\operatorname{Der}^{n}(A / k)$ for all $n$, then $A$ is integrally closed.

Proof. The proof is by contradiction. Let $Q$ denote the quotient field of $A$, and let $\bar{A}$ denote the integral closure of $A$ in $Q$. We assume $A \neq \bar{A}$. Thus, the conductor $C=A: \bar{A}$ is a proper ideal in $A$, i.e., $0<C<A$. Let $p$ be an associated prime of $C$. It follows from [7, Corollary, p. 169] that $C$ is a differential ideal in $A$. Since $p$ is an associated prime of $C, p$ is a differential prime ideal in $A[8$, Theorem 1]. Thus by hypothesis, $p$ is a minimal prime ideal of $A$.

If $R$ is any $k$-algebra, we shall denote by $D^{1}(R / k)$ the $R$-module of first order differentials of $R$ over $k$ (see [5]).

Now consider the local ring $A_{p}$. The integral closure $\bar{A}_{p}$ of $A_{p}$ in $Q$ is a finitely generated $A_{p}$-module and hence a semilocal ring. Let $\left\{\bar{p}_{1}, \cdots, \bar{p}_{m}\right\}$ denote the maximal ideals of $\bar{A}_{p}$. Since $\left(\bar{A}_{p}\right)_{\tilde{p}_{i}}=V_{i}$ is a discrete rank one valuation ring, [5, Theorem $\left.3^{\prime}\right]$ implies that $D^{1}\left(V_{i} / k\right)$ is a free $V_{i}$-module of rank $r$. Here $r$ is the transcendence degree of $A$ over $k$. It follows that $D^{1}\left(\bar{A}_{p} / k\right)$ is a projective $\bar{A}_{p}$-module of rank $r$. Thus, since $\bar{A}_{p}$ is semilocal, $D^{1}\left(\bar{A}_{p} / k\right)$ is a free $\bar{A}_{p}$-module of rank $r$.

Let $d: A_{p} \rightarrow D^{1}\left(A_{p} / k\right)$ and $\bar{d}: \bar{A}_{p} \rightarrow D^{1}\left(\bar{A}_{p} / k\right)$ denote the canonical $k$ derivations of $A_{p}$ and $\bar{A}_{p}$ into $D^{1}\left(A_{p} / k\right)$ and $D^{1}\left(\bar{A}_{p} / k\right)$ respectively. If $K(A / p)$ denotes the quotient field of $A / p$, then $K(A / p)$ has transcendence degree $r-1$ over $k$. Thus, there exist elements $\alpha_{1}, \cdots, \alpha_{r-1} \in A-p$ such that $K(A / p)$ is a separable algebraic extension of $k\left(\bar{\alpha}_{1}, \cdots, \bar{\alpha}_{r-1}\right)$. Here $\bar{\alpha}_{i}$ of course denotes the image of $\alpha_{i}$ in $A / p$. We note that $F=k\left(\alpha_{1}, \cdots, \alpha_{r-1}\right)$ is a field contained in $A_{p}$.

By [9, Theorem 18, p. 45], there exists a $\beta \in \bigcap_{i=1}^{m} \bar{p}_{i}$ such that $\beta$ generates the maximal ideal of each $V_{i}$. From the proof of [5, Theorem $3^{\prime}$ ], we have the following short exact sequence of $V_{i}$-modules:

$$
0 \rightarrow V_{i} \otimes_{F} D^{1}(F / k) \rightarrow D^{1}\left(V_{i} / k\right) \rightarrow D^{1}\left(V_{i} / F\right) \rightarrow 0 .
$$

It easily follows from $(1)$ that $\left\{d_{i}(\beta), d_{i}\left(\alpha_{1}\right), \cdots, d_{i}\left(\alpha_{r-1}\right)\right\}$ is a free $V_{i}$-basis of $D^{1}\left(V_{i} / k\right)$. Here $d_{i}: V_{i} \rightarrow D^{1}\left(V_{i} / k\right)$ is the canonical derivation. An 
application of Nakayama's lemma shows that $\left\{\bar{d}(\beta), \bar{d}\left(\alpha_{1}\right), \cdots, \bar{d}\left(\alpha_{r-1}\right)\right\}$ is a free basis of $D^{1}\left(\bar{A}_{p} / k\right)$.

Let $\left\{\Psi_{0}, \Psi_{1}, \cdots, \Psi_{r-1}\right\} \subset \operatorname{Hom}_{\bar{A}_{p}}\left(D^{1}\left(\bar{A}_{p} / k\right), \bar{A}_{p}\right)$ be a dual basis to $\left\{\bar{d}(\beta), \bar{d}\left(\alpha_{1}\right), \cdots, \bar{d}\left(\alpha_{r-1}\right)\right\} \subset D^{1}\left(\bar{A}_{p} / k\right)$. Then setting $\delta_{i}=\Psi_{i} \circ \bar{d}$, we get $r$ $k$-derivations of $\bar{A}_{p}$ such that $\delta_{0}(\beta)=\delta_{i}\left(\alpha_{i}\right)=1, \delta_{0}\left(\alpha_{i}\right)=\delta_{i}(\beta)=0$ and $\delta_{i}\left(\alpha_{j}\right)=0$ if $i \neq j$. Since $\operatorname{Hom}_{I_{p}}\left(D^{1}\left(\bar{A}_{p} / k\right), \bar{A}_{p}\right) \cong \operatorname{Der}^{1}\left(\bar{A}_{p} / k\right)$, we see that $\left\{\delta_{0}, \delta_{1}, \cdots, \delta_{r-1}\right\}$ is a free $\bar{A}_{p}$-module basis for $\operatorname{Der}^{1}\left(\bar{A}_{p} / k\right)$. Now if $\varphi \in$ $\operatorname{Der}^{1}\left(A_{p} / k\right)$, then by [7, Theorem] $\varphi$ extends uniquely to $\hat{\varphi} \in \operatorname{Der}^{1}\left(\bar{A}_{\mathcal{D}} / k\right)$. Thus, there exist $a_{0}, \cdots, a_{r-1} \in \bar{A}_{p}$ such that

$$
\hat{\varphi}=a_{0} \delta_{0}+a_{1} \delta_{1}+\cdots+a_{r-1} \delta_{r-1} \text {. }
$$

We wish to characterize $a_{0}$.

Now $d$ when restricted to $A_{p}$ gives a $k$-derivation of $A_{p}$ into $D^{1}\left(\bar{A}_{p} / k\right)$. Hence, by the universal mapping property of $D^{1}\left(A_{p} / k\right)$, there exists a unique $A_{p}$-module homomorphism $\sigma: D^{1}\left(A_{p} / k\right) \rightarrow D^{1}\left(\bar{A}_{p} / k\right)$ such that $\sigma \circ d=\left.d\right|_{A_{p}}$. Since $d\left(\alpha_{i}\right) \in D^{1}\left(A_{p} \mid k\right), d\left(\alpha_{i}\right) \in \operatorname{Im} \sigma$. Thus, we can write

$$
\operatorname{Im} \sigma=M \dot{d}(\beta) \oplus A_{p} d\left(\alpha_{1}\right) \oplus \cdots \oplus A_{p} d\left(\alpha_{r-1}\right)
$$

for some $A_{p}$-submodule $M \subset \bar{A}_{p}$. If $T=\operatorname{ker} \sigma$, then

$$
D^{1}\left(A_{p} / k\right) \otimes_{A_{D}} Q \cong D^{1}(Q / k) \cong D^{1}\left(\overline{A_{p}} / k\right) \otimes_{A_{D}} \overline{A_{D}}
$$

implies that $T$ is a torsion submodule of $D^{1}\left(A_{p} / k\right)$. Thus, we have

$$
\begin{aligned}
\operatorname{Hom}_{A_{p}}\left(\operatorname{Im} \sigma, A_{p}\right) & \cong \operatorname{Hom}_{A_{p}}\left(D^{1}\left(A_{p} / k\right) / T, A_{p}\right) \\
& \cong \operatorname{Hom}_{A_{p}}\left(D^{1}\left(A_{p} / k\right), A_{p}\right) \cong \operatorname{Der}^{1}\left(A_{p} / k\right) .
\end{aligned}
$$

Now, if $M=(0)$, then $\operatorname{Im} \sigma$ is a free $A_{p}$-module. Hence by (2), $\operatorname{Der}^{1}\left(A_{p} / k\right)$ is a free $A_{p}$-module. But then [3, Theorem 1] implies that $A_{p}$ is normal which is a contradiction. Thus, $M$ is a nonzero submodule of $\bar{A}_{p}$.

We next note that if $\varphi \in \operatorname{Der}^{1}\left(A_{p} / k\right)$, and we write $\hat{\varphi}=a_{0} \delta_{0}+a_{1} \delta_{1}+\cdots+$ $a_{r-1} \delta_{r-1}$, then $a_{0} M \leqq A_{p}$. If $a_{0} M=A_{p}$, then $M$ is a free $A_{p}$-module. Consequently, $\operatorname{Im} \sigma$ is free, and we again reach a contradiction. Thus, $a_{0} M \leqq$ $p A_{p}$, the maximal ideal of $A_{p}$.

Now let $\left\{z_{1}, \cdots, z_{l}\right\}$ be a minimal basis for the maximal ideal $p A_{p}$. By $[5,(\mathrm{G})]$ the following sequence of $A_{p} / p A_{p}$-modules is exact:

(3) $0 \rightarrow p A_{p} / p^{2} A_{p} \rightarrow\left(A_{p} / p A_{p}\right) \otimes_{A_{p}} D^{1}\left(A_{p} / k\right) \rightarrow D^{1}\left(\left(A_{p} / p A_{p}\right) / k\right) \rightarrow 0$.

Since $A_{p} / p A_{p} \cong K(A / p)$ is a separable algebraic extension of $F,(3)$ implies that $D^{1}\left(A_{p} / k\right)$ is generated as an $A_{p}$-module by $\left\{d\left(z_{1}\right), \cdots, d\left(z_{l}\right)\right.$, $\left.d\left(\alpha_{1}\right), \cdots, d\left(\alpha_{r-1}\right)\right\}$. Thus, $\left\{\delta_{0}\left(z_{1}\right), \cdots, \delta_{0}\left(z_{l}\right)\right\}$ generate $M$. 
The argument from this point on is essentially that found in [4] applied to the $\operatorname{Der}^{1}\left(A_{p} / F\right)$-components of $\operatorname{Der}^{1}\left(A_{p} / k\right)$. If $v_{i}$ denotes the valuation on $V_{i}$, then $v_{i}\left(\delta_{0}\left(z_{j}\right)\right)=v_{i}\left(z_{j}\right)-1$ for all $j=1, \cdots, l$ and $i=1, \cdots, m$. Thus if $\varphi \in \operatorname{Der}^{1}\left(A_{p} / k\right)$ and $\hat{\varphi}=a_{0} \delta_{0}+\cdots+a_{r-1} \delta_{r-1}$, then $a_{0} \in \bigcap_{i=1}^{m} \bar{p}_{i}$. If $I=\left\{a_{0} \in \bar{A}_{p} \mid a_{0} \delta_{0}+\cdots+a_{r-1} \delta_{\tau-1} \in \operatorname{Der}^{1}\left(A_{p} \mid k\right)\right.$ for some $\left.a_{i} \in \overline{A_{p}}\right\}$, then $I V_{1}=\left(\beta^{f}\right)$ for some integer $f \geqq 1$. Set $C \cdot V_{1}=\left(\beta^{c}\right)$ and find $y \in C$ such that $v_{1}(y)=c$. Choose an integer $N$ sufficiently large so that $f N>c$. Then $y \delta_{0}^{N} \in \operatorname{Der}^{N}\left(A_{p} / k\right)$ but $y \delta_{0}^{N} \notin \operatorname{der}^{N}\left(A_{p} / k\right)$. Thus hypothesis (2) in the theorem is contradicted and the proof is complete.

We can now prove the main result of this paper.

THEOREM 2. Let $A=k\left[x_{1}, \cdots, x_{t}\right]$ be a finitely generated integral domain over a field $k$ of characteristic zero. Then $A$ is regular if and only if (1) no nonminimal prime ideal $q \subseteq A$ is differential, and (2) $\operatorname{der}^{n}(A / k)=$ $\operatorname{Der}^{n}(A / k)$ for all $n$.

Proof. If $A$ is regular, then condition (1) follows from [8, Theorem 3]. Condition (2) follows from [2, Theorem 16.11, 2].

So conversely suppose $A$ satisfies conditions (1) and (2). By Theorem 1, $A$ is an integrally closed domain. Suppose $q$ is a prime ideal of $A$ of height one. Then $A_{a}$ is a discrete rank one valuation ring and hence a regular local ring. Let us assume that $A_{q}$ is a regular local ring for all primes $q$ having height less than $k$. Here $1 \leqq k<r$ the transcendence degree of $A$ over $k$. Now suppose $q$ is a prime ideal of height $k+1$ in $A$. Then by the induction hypothesis every proper localization of $A_{q}$ is a regular local ring. If we assume $A_{a}$ itself is not regular, then it follows from [8, Theorem 5] that $q A_{q}$ is a differential ideal in $A_{a}$. But, $q$ is a nonminimal prime of $A$ and hence by hypothesis is not differential in $A$. Thus $q A_{q}$ is not differential in $A_{q}$. Consequently, $A_{Q}$ must be regular and the proof is complete.

\section{REFERENCES}

1. N. Bourbaki, Eléments de mathématique. Fasc. XXVII. Algèbre commutative. Chap. 1: Modules plats. Chap. 2: Localisation, Actualités Sci. Indust., no. 1290, Hermann, Paris, 1961. MR 36 \#146.

2. A. Grothendieck, Eléments de géométrie algébrique, Inst. Hautes Etudes Sci. Publ. Math. Nos. 4-32 (1960-67). MR 29 \#1210; 30 \#3885; 33.\#7330; 36 \#177a,b,c; 36 \#178; 39 \#220.

3. J. Lipman, Free derivation modules on algebraic varieties, Amer. J. Math. 87 (1965), 874-898. MR 32 \#4130.

4. K. Mount and O. E. Villamayor, On a question of Y. Nakai, Osaka J. Math. (to appear).

5. Y. Nakai, On the theory of differentials in commutative rings, J. Math. Soc. Japan 13 (1961), 63-84. MR 23 \#A2437. 
6. Y. Nakai, Higher order derivations. I, Osaka J. Math.7(1970), 1-27. MR 41 \#8404.

7. A. Seidenberg, Derivations and integral closure, Pacific J. Math. 16 (1966), 167173. MR 32 \#5686.

8. - Differential ideals in rings of finitely generated type, Amer. J. Math. 89 (1967), 22-42. MR 35 \#2902.

9. O. Zariski and P. Samuel, Commutative algebra. II, University Series in Higher Math., Van Nostrand, Princeton, N.J., 1960. MR 22 \#11006.

Department of Mathematics, Michigan State University, East Lansing, Michigan 48823 